\title{
Philosophiques
}

\section{Wittgenstein et Ortega sur les lois logiques de base}

\section{Patrice Philie}

Volume 42, numéro 2, automne 2015

URI : https://id.erudit.org/iderudit/1034749ar

DOI : https://doi.org/10.7202/1034749ar

Aller au sommaire du numéro

Éditeur(s)

Société de philosophie du Québec

ISSN

0316-2923 (imprimé)

1492-1391 (numérique)

Découvrir la revue

Citer ce document

Philie, P. (2015). Wittgenstein et Ortega sur les lois logiques de base.

Philosophiques, 42(2), 391-399. https://doi.org/10.7202/1034749ar d'utilisation que vous pouvez consulter en ligne.

https://apropos.erudit.org/fr/usagers/politique-dutilisation/ 


\title{
Wittgenstein et Ortega sur les lois logiques de base
}

\author{
PATRICE PHILIE \\ Université d'Ottawa
}

Le livre de Kevin Mulligan ${ }^{1}$ qui fait l'objet de cette disputatio porte sur Wittgenstein et ses liens avec les traditions austro-allemandes provenant de Bolzano et Brentano. Ces deux penseurs sont à l'origine, entre autres, des courants phénoménologiques et de la psychologie de la Gestalt. Selon Mulligan, l'influence s'étend même jusqu'aux confins du Cercle de Vienne (donc très près de Wittgenstein, conceptuellement et au sens propre). La présente contribution se concentrera sur le dernier chapitre du livre, qui porte sur les certitudes. Tout comme dans les chapitres précédents, Mulligan nous offre ici une analyse comparative très riche entre les travaux des penseurs issus de la tradition austro-allemande et les écrits de Wittgenstein. Tout d'abord, et crucialement, les deux accordent un rôle central aux descriptions. Au premier chapitre de Wittgenstein et la philosophie austro-allemande, Mulligan décrit bien, pour ainsi dire, les similitudes et les différences entre les descriptions de Wittgenstein (seconde manière) et celles de ses prédécesseurs phénoménologues. La différence qui m’interpelle le plus est soulignée par Mulligan au premier chapitre: "Alors que ses prédécesseurs conçoivent la description comme portant avant tout sur la complexité des états et des épisodes mentaux psychologiques ainsi que sur leurs objets, Wittgenstein considère qu'il décrit le langage et la façon dont on emploie les mots ${ }^{2} »(47)$. Cela illustre selon moi la différence fondamentale entre les deux types de description: alors que Wittgenstein tente en fait d'expulser, ou tout du moins de minimiser, le rôle des descriptions qui réfèrent à des états mentaux ou "internes ", les phénoménologues n'ont aucun scrupule à explorer et à décrire notre vie intérieure, notre vie mentale. $\mathrm{Ou}$, pour le dire peut-être plus précisément, Wittgenstein croit qu'il n'est pas souhaitable de faire appel aux états et épisodes mentaux lorsque l'on tente de faire la lumière sur les concepts qui le préoccupent, notamment ceux de signification, de vouloir-dire, de connaissance, et de certitude - et cela est à contraster avec ce que font les phénoménologues issus de la tradition austro-allemande telle que comprise par Mulligan.

Dans De la certitude ${ }^{3}$, Wittgenstein tente justement - de façon presque obsessive - de décrire ce que signifie être certain de savoir quelque

1. Wittgenstein et la philosophie austro-allemande, Vrin, $20 \mathrm{I} 2$.

2. Les numéros de page font référence à Wittgenstein et la philosophie austro-allemande, sauf indication contraire.

3. Gallimard, 2006. Traduction de Danièle Moyal-Sharrock. Le livre a été publié pour la première fois en I969. Selon les éditeurs, Wittgenstein a écrit ces remarques entre I 949 et I95 I.

PHILOSOPHIQUES 42/2 - Automne 2015, p. 391-399 
chose, et cela l'amène évidemment à examiner les concepts connexes de doute, de connaissance, et de conviction. Cet examen met en lumière plusieurs idées évocatrices et bien connues de cet ensemble de remarques, comme la fin de la justification, la fluidité des propositions empiriques, le rôle des propositions charnières, et ainsi de suite. Les philosophes étudiés par Mulligan au chapitre VIII — dont le plus intéressant, selon moi, est José Ortega y Gasset — arrivent à des positions semblables à celles de Wittgenstein, et les similitudes qu'il souligne sont surprenantes et même stimulantes, ne serait-ce que pour éclairer les positions de Wittgenstein, qui peuvent parfois être nébuleuses telles qu'elles sont présentées. Mais avant de commencer l'analyse critique du chapitre VIII du livre de Mulligan, il est nécessaire de se pencher sur ce qu'est, en quelque sorte, De la certitude.

Cet ensemble de remarques est en fait tiré de différents manuscrits écrits dans les deux dernières années de sa vie. Il est important de noter une évidence que nous sommes prompts à oublier, étant donné le mode de présentation des remarques sous la forme du livre édité par Anscombe et von Wright: ces remarques sont en fait une collation d'un premier jet de remarques portant sur un ensemble de thèmes connexes. L'ordre de présentation est temporel, et ne suit donc pas un ordre logique (en ce sens cet ordre est «arbitraire »), ainsi bien sûr que la numérotation, qui ne provient pas de Wittgenstein lui-même. Il écrivait des remarques sur ces sujets. Parfois, les remarques suivent un ordre logique. Parfois, elles sautent du coq à l'âne. Toujours, elles reviennent à un examen de thèmes reliés, de thèmes épistémologiques. Wittgenstein n'a fait aucun travail de révision sur ces remarques. Il n'a rien fait pour en sélectionner certaines, aucune indication n'est donnée sur les remarques qu'il trouve plus importantes, moins importantes. Aucune indication non plus n'est fournie sur un éventuel ordre de présentation. En ce sens, De la certitude s'apparente beaucoup plus aux Last Writings on the Philosophy of Psychology ${ }^{4}$ qu'à, par exemple, les Fiches ${ }^{5}$ ou même les Remarques sur la philosophie de la psychologie ${ }^{6}$, qui sont basés sur des copies dactylographiées et ont donc fait l'objet d'une sélection et d'un travail de révision par Wittgenstein lui-même. Nous sommes loin, très loin, d'un ouvrage poli et réfléchi comme Les recherches philosophiques ${ }^{7}$. De la certitude est un ensemble de remarques exploratoires, le fruit brut d'une réflexion préliminaire sur le sujet, un peu comme les Carnets $^{8}$ (dont il voulait d'ailleurs qu'ils soient détruits). Il n'est donc pas surprenant qu'il se contredise dans cet ouvrage et qu'il semble souvent hésiter entre diverses

4. Traduction française en deux volumes chez T.E.R. Volume I, Études préparatoires à la seconde partie des Recherches Philosophiques (I985). Volume 2, L'intérieur et l'extérieur. Derniers écrits sur la philosophie de la psychologie (2000).

5. Gallimard (2008).

6. T.E.R. (I994).

7. Gallimard (2004).

8. Gallimard (I97I). 
positions: il est en mode "recherche». Une quarantaine de jours avant sa mort, il écrivait encore dans De la certitude:

400. Ici, j'ai tendance à me battre contre des moulins à vent, car je n'arrive pas encore à dire ce que je veux vraiment dire.

Il faut ainsi prendre les remarques pour ce qu'elles sont: celles d'un génie philosophique en train de réfléchir à un ensemble de problèmes reliés et qui écrit les pensées lui venant à l'esprit, sans encore tenter de former un tout cohérent, encore moins un «livre» destiné à être publié. Cependant, le fait que le livre tel que nous le connaissons possède, en apparence, la même forme qu'un ouvrage achevé comme les Recherches - numérotation des remarques, apparence d'un tout continu — tend à aplanir les différences fondamentales entre les deux. La première remarque de De la certitude n'est pas la première remarque d'un livre que Wittgenstein a écrit, et la dernière remarque n'est pas la conclusion de ce «livre» qui n'en est pas un au sens strict.

Cela dit, il est indéniable qu'une lecture attentive de ces remarques permet de dégager certains thèmes cruciaux, thèmes qui sont repris par Mulligan dans son étude. En premier lieu, Mulligan souligne le cadre théorique semblable des deux types de description (wittgensteiniennes et celles de la tradition austro-allemande) lorsque celles-ci traitent de la certitude: par exemple, les deux types font appel aux certitudes primitives, à la distinction entre les certitudes objectives et les certitudes subjectives, au fait que nos certitudes forment un "système ", etc. De mon point de vue, les positions de Wittgenstein à ce sujet sont assez familières; il n'y a là aucune surprise de voir que Wittgenstein traite de ces thèmes, et de cette façon, dans ses remarques. Cependant, il est plutôt surprenant de constater, grâce à Mulligan, à quel point les recherches d'Ortega arrivent à des conclusions similaires. Que démontre cette similitude de résultats? Probablement que les grandes lignes qu'ils dégagent de leurs analyses respectives correspond à une façon naturelle de voir les choses: il y a un groupe de "propositions " qui jouent un rôle spécial dans nos vies. Elles ont la forme de propositions dites "normales", certaines sont même de forme empirique, et pourtant elles ne sont pas comme "le chat est sur le paillasson». Elles sont certaines, et elles forment l'arrière-plan de nos croyances ordinaires. Considérées dans leur ensemble, elles forment même un système.

Voilà pour le tour d'horizon et la mise en contexte. J'aimerais dans ce qui suit examiner de plus près deux aspects de l'analyse de Mulligan. Le premier aspect portera sur un type de certitude primitive et objective qui n'est pas à proprement parler mentionné dans le livre, c'est-à-dire les lois logiques fondamentales. Le deuxième portera sur l'aspect normatif des certitudes, un thème important qui occupe une place de choix à la fin du chapitre de Mulligan.

Les lois logiques fondamentales que j'ai en tête sont celles qui déterminent le raisonnement correct, que l'on parle ici du raisonnement correct 
du discours de tous les jours, ou des divers discours théoriques. Je ne sais pas quelles sont précisément ces lois logiques, mais pour des raisons que je ne peux discuter ici, la liste, si elle existe, inclut très certainement le modus ponens. Cette loi est une certitude primitive au sens d'Ortega, dont il dit à leur propos "que nous comptons sur elles - toujours, sans interruption" (I8I-I82). Elles le sont aussi pour Wittgenstein - le modus ponens est "fermement établi" » pour nous. Il est étonnant que Wittgenstein ne parle pas explicitement des lois logiques dans De la certitude, mais cependant il fait souvent allusion aux "propositions de la logique » et celles-ci incluent, je crois, les lois logiques. En tout les cas, il est tentant de lire $\$ 152$ de De la certitude à la lumière d'une loi comme le modus ponens:

I 52. Les propositions qui sont solidement fixées pour moi, je ne les apprends pas explicitement. Je peux les découvrir ultérieurement comme l'axe autour duquel pivote un corps. L'axe n'est pas fixé dans le sens où il serait maintenu par quelque chose, c'est le mouvement autour de lui qui détermine son immobilité.

Cette image est différente des métaphores géologiques habituellement utilisées par Wittgenstein et Ortega, et peut-être plus précise en cela qu'elle porte moins à voir les choses de façon "fondationnaliste" que les métaphores géologiques - surtout celle du bedrock (le "roc dur" dans la traduction française). Et lorsque Wittgenstein note le point important que nous n'apprenons pas ces propositions, cela me fait penser à ce que le moraliste français Vauvenargues écrivait deux siècles avant Wittgenstein: "Les choses que l'on sait le mieux sont celles qu'on n'a pas apprises » (Réflexions et Maximes, I746).

Il me semble clair que le modus ponens satisfait ces conditions. Un enfant de 3 ans «maîtrise» le modus ponens et les autres lois logiques fondamentales, mais elles n'ont pas été apprises, et surtout pas explicitement. Cependant, c'est une des choses que, dirait Vauvenargues, l'on sait le mieux: tout le monde «suit» cette règle. Pourtant, peu d'entre nous sont capables de rendre ce savoir explicite par eux-mêmes: il nous a tous fallu suivre un cours de logique pour être conscient de la loi logique du modus ponens selon laquelle il est permis de détacher le conséquent d'un énoncé conditionnel lorsque l'antécédent est satisfait:

\section{Si : $(p \rightarrow q) \& p$}

Alors: $q$

Si on y pense, la formulation de ce principe est assez compliquée et présuppose un arrière-plan de connaissances assez substantiel - cependant, en pratique, cette règle est suivie par tous les agents doués de raison. Et

9. De la certitude, $\mathbb{S}$ I52, cité par Mulligan à la page I 82. Moyal-Sharrock traduit de son côté feststehen par "solidement fixé», comme le montrera la citation complète de la remarque. 
comme le dit Wittgenstein, nous pouvons découvrir la règle ultérieurement, même si dans les faits nous l'avons en quelque sorte «internalisée » avant de la découvrir. Et cette internalisation est nécessaire pour la rationalité au sens où elle fait partie du corps de propositions qui soutiennent le reste, sans elles-mêmes être soutenues - elles sont primitives.

Les lois logiques fondamentales sont aussi objectives au sens de Mulligan. De la façon dont je comprends la distinction entre certitude subjective et certitude objective, la certitude subjective correspond à un sentiment de conviction tandis que la certitude objective correspond à l'idée selon laquelle l'erreur est (logiquement?) exclue. «Je suis certain que le chat est sur le paillasson" serait un exemple de certitude subjective. Que le chat soit sur le paillasson est peut-être intéressant et même important dans un certain contexte, mais la proposition n'est pas une certitude objective, car il est possible de se tromper au sujet du fait qu'il y a un chat sur le paillasson tandis qu'il n'est pas possible que de $((p \rightarrow q) \& p)$ il ne s'ensuive pas que $q$.

Ainsi, les lois logiques sont des certitudes primitives et objectives au sens d'Ortega et de Wittgenstein. Elles ne sont pas apprises explicitement et font partie des propositions charnières qui déterminent ce que nous disons et pensons, et comment nous pensons. À mon avis, l'objectif de Wittgenstein dans De la certitude est de faire la lumière sur le rôle de ces propositions dans nos vies. Elles sont déterminantes et définissent, en quelque sorte, notre forme de vie. Je ne crois pas, contrairement à une opinion assez répandue, que Wittgenstein tente ici de «répondre " au scepticisme. Si je peux me permettre d'oser un peu, Mulligan serait peut-être d'accord avec moi sur ce sujet - la façon dont il traite la position de Wittgenstein laisse croire que lui aussi est d'avis que Wittgenstein explore le statut des propositions charnières et tente de les situer dans ce que je vais appeler, faute de mieux, notre "schème conceptuel ". Selon moi, lire De la certitude de cette façon est plus riche et stimulant - il s'agirait en fait ici d'explorer la condition humaine, rien de moins - que de le réduire à une réponse à un problème philosophique (bien qu'il s'agisse aussi de cela ${ }^{10}$ ). Cela le rapprocherait aussi des intérêts d'Ortega, qui se situent au-delà de l'élucidation d'un problème purement philosophique n'ayant qu'un effet minime sur notre compréhension la condition humaine.

Ortega, en effet - et cela m'amène à la deuxième partie de mon analyse - est préoccupé par des enjeux qui dépassent le cadre souvent très technique et spécialisé de la philosophie (surtout analytique) du milieu du $\mathrm{XX}^{\mathrm{e}}$ siècle. Il étudie notre rapport aux normes politiques, éthiques, sociales, et religieuses, pour ne nommer que celles-là. Il croit aussi que ces normes forment un ensemble de certitudes primitives collectives et constituent un

10. D'une façon perverse, car Wittgenstein «répond" au problème du scepticisme en tentant de le démasquer comme étant un pseudo-problème, et non pas en y fournissant une «solution» au sens traditionnel. 
«pouvoir social» (2I 5 ) - on croirait presque lire Foucault. Wittgenstein ne discute pas de ces normes. Il est plutôt préoccupé par des normes encore plus fondamentales à mon avis, car, sans celles-ci, il serait impossible d'articuler ou même d'internaliser une norme éthique ou politique. Je dois admettre que je ne comprends pas ce que serait une certitude primitive politique - de la façon dont je conçois une telle certitude, une norme politique est toujours susceptible d'être révisée. Mais passons outre, puisque Ortega mentionne que les normes linguistiques sont aussi des certitudes primitives - je crois qu'on peut comprendre les normes linguistiques d'Ortega comme étant similaires aux règles et propositions logiques de Wittgenstein dans $D e$ la certitude. La position d'Ortega à leur sujet est drôlement évocatrice pour un lecteur de Wittgenstein. Je cite Mulligan à propos d'Ortega:

Il est fondamentalement erroné [selon Ortega] de comprendre les usages, en particulier «l'immense système des usages verbaux» qu'est un langage, en termes de régularités ou de fréquences comportementales [...] Le rejet par Ortega de ce qui a été appelé le « régularisme» au sujet des règles est combiné avec le rejet de la thèse selon laquelle ce qui rend une règle liante est le fait qu'une communauté y adhère, ce qui a été appelé le "communautarisme » $[. .$. Puisque les usages sont primitivement certains, nous ne les comprenons pas, ils sont inintelligibles; bien que nous comprenions ce que nous avons à faire, nous nous conformons à un usage, parce que cet usage est ce qui se fait (2 I 52I6).

Ce passage montre à quel point la position d'Ortega sur le principe de "suivre une règle» est proche de celle de Wittgenstein. Mais cela seulement à la condition de ne pas souscrire à l'idée, encore assez répandue, selon laquelle l'auteur des Recherches philosophiques est un communautariste pour ce qui est de suivre une règle ${ }^{11}$. Je n'ai pas le temps d'en faire la démonstration ici, ce n'est pas le propos, mais je suis d'avis que la position de Wittgenstein sur ce que c'est que suivre une règle est beaucoup plus subtile, et surtout, plus crédible que la lecture communautariste ne le laisse entendre. En ce sens, Wittgenstein est très proche d'Ortega.

Mais ramenons ces considérations aux lois logiques fondamentales. Il me semble clair, et surtout raisonnable, de croire que ce qui rend le modus ponens «correct» n'est pas le simple fait que nous suivons tous cette règle. Cela le rendrait optionnel en quelque sorte, au sens où il serait possible, en théorie, que nous n'y adhérions pas. À mon avis, nous n'avons pas le choix d'adhérer ou non à cette norme, et le contraire n'est même pas imaginable. La régularité est une caractéristique des normes, tout comme le fait que tous $\mathrm{y}$ adhèrent. Mais celles-ci ne peuvent faire que les normes fondamentales

11. Kripke, dans Règles et langage privé. Introduction au paradoxe de Wittgenstein (Seuil, 1996), chapitre III, est le locus classicus. Le débat sur l'interprétation correcte des passages sur ce qu'est «suivre une règle» dans les Recherches philosophiques est très complexe. Il est impossible ici de s'étendre davantage sur ce sujet. 
soient correctes - elles ne sont que la façon dont les normes agissent en tant que normes.

J'aimerais en profiter ici pour souligner le caractère fortement normatif du système formé par les certitudes primitives que sont les propositions logiques et les règles de base chez Wittgenstein. Mulligan semble hésiter à attribuer à Wittgenstein la position selon laquelle les certitudes primitives sont pour ce dernier des normes (2I4). Son hésitation est basée sur deux observations. La première est qu' "aucune attitude ou disposition psychologique ou mentale n'est une norme, pas plus que ne l'est l'attitude ou la disposition de compter pratiquement sur quelque chose» (2I4). Je comprends l'objection de Mulligan: comment un simple état mental comme la certitude peut-il constituer une norme? Il semble constitutif de ce qu'est une norme que celle-ci doive en quelque sorte se situer hors de la sphère purement subjective qu'est un sentiment de conviction. Ainsi, ma certitude que le chat est sur le paillasson n'est pas une norme. Cependant, s'il s'agit d'une certitude primitive objective comme «il y a un monde extérieur ", ne peut-on pas attribuer à cette proposition le statut de norme, étant donné qu'elle sous-tend, en quelque sorte, des propositions empiriques normales comme «le chat est sur le paillasson»? Il semble que selon Wittgenstein de telles propositions soient des normes, compte tenu de la remarque suivante de De la certitude:

I67. Il est clair que toutes nos propositions empiriques n'ont pas le même statut, étant donné qu'on peut fixer une de ces propositions, et d'une proposition empirique en faire une norme de description.

La distinction entre proposition empirique potentiellement normative et proposition empirique pour laquelle le statut de norme ne semble pas possible n'est-elle pas la même que la distinction entre certitude subjective et certitude objective? Ou, à tout le moins, ne pourrait-on pas dire qu'une norme de description consiste en un sous-ensemble des certitudes objectives? Si une certitude est "objective", cela veut dire qu'elle n'est pas purement le produit d'un sentiment de conviction. Cette certitude est en quelque sorte indépendante de ce que j'en pense - elle est établie objectivement. Ainsi, une telle certitude n'est pas purement une attitude ou une disposition psychologique ou mentale, et peut éventuellement constituer une norme. Je dois admettre que je ne sais pas - en cela je ne suis certainement pas le seul - ce qu'est une norme. La question est, à mon avis, ouverte. Indépendamment de ces considérations, n'est-il pas clair que le modus ponens est une norme?

J'aimerais terminer cette discussion en examinant un passage fort intéressant du livre de Mulligan, dans lequel il avance une autre considération pour résister à l'idée que les certitudes primitives wittgensteiniennes sont des normes. Selon lui, affirmer que les propositions charnières de Wittgenstein dans De la certitude sont des normes revient à nier que «la description de 
notre rapport aux normes doit bien souvent faire appel à la certitude primitive et rend le fait que notre rapport aux normes politiques et éthiques ne ressemble en rien à notre rapport aux normes épistémiques difficile à expliquer» (2I4). Il y a deux choses que j'aimerais relever dans ce passage. Premièrement, Mulligan nous rappelle ici le caractère absolument fondamental des normes épistémiques et de représentation. En effet, tout ce que nous disons, tout ce que nous soutenons, est tributaire de ces normes. Cela inclut bien sûr notre discours sur les normes politiques et éthiques, qui dépend, pour leur intelligibilité même, des normes fondamentales; des propositions charnières sont présupposées dans ce discours, et parmi elles le modus ponens doit occuper une place de choix. Ce sont ces normes fondamentales qui intéressent Wittgenstein. Elles sont normatives en ce qu'elles déterminent ce que nous disons - nous devons, par exemple, obéir au modus ponens, et en ce sens il est clair qu'il s'agit d'une norme. Mais c'est aussi un genre de norme particulier, une norme enfouie profondément dans l'ensemble de propositions auxquelles nous adhérons, au sens où nous n'en sommes pas conscients de la même façon que nous pouvons être «conscients» que la torture est moralement inacceptable ou que l'extrême droite est un courant politique déviant. Cependant, contrairement à Mulligan, je ne crois pas que cet aspect des certitudes primitives wittgensteiniennes doive nous empêcher de considérer celles-ci comme des normes.

La deuxième chose dont j'aimerais discuter dans ce passage est l'idée selon laquelle notre rapport avec ces certitudes primitives épistémiques et représentationnelles est "difficile à expliquer». En effet. Le contraste ici avec les normes épistémiques et politiques est clair: un peu (parfois beaucoup) de réflexion et d'examen de soi suffit en principe à articuler les normes de ce genre, auxquelles nous adhérons. À première vue, articuler une norme politique ne présuppose pas une norme politique - à tout le moins, l'articulation d'une norme politique ne présuppose pas que nous y adhérons. Je peux articuler les normes de l'extrême droite, ou bref, j'imagine que je pourrais le faire mais je n'y adhère très certainement pas. Cependant, comment articuler les normes qui régissent tout discours sans présupposer ces normes, puisque cette articulation est elle-même un discours? C'est impossible, et c'est là un des défis posés dans De la certitude. Ainsi, un discours descriptif sur les normes épistémiques et les normes de représentation semble voué à l'échec - à tout le moins si nous exigeons d'une description qu'elle ne fasse pas appel à ce qu'elle décrit. Si nous ne pouvons articuler ces «normes» de façon satisfaisante, peut-on dire qu'elles en sont? Et surtout, si nous ne pouvons rendre compte de notre rapport avec ces supposées normes, comment pouvons-nous affirmer que nous entretenons un rapport normatif avec ces propositions? C'est de cette façon que je comprends l'interrogation de Mulligan.

Je crois qu'il faut séparer deux problèmes: d'un côté il y a le fait qu'une description des normes de base présuppose l'utilisation de ces normes. Le 
problème engendré par cet état de choses est le suivant: il semble que cela rende impossible la contemplation, pour ainsi dire, de la norme d'un point de vue purement objectif, c'est-à-dire de "l'extérieur », comme il est possible de le faire pour une norme, disons, politique. Bref, cela rend problématique la question de notre rapport aux normes qui régissent tout discours. Ce premier problème donne naissance au deuxième, que l'on pourrait formuler ainsi: si tout discours présuppose un ensemble de normes $N$, comment allons-nous rendre compte de la justification de ces normes? En effet, une telle justification prendra nécessairement la forme d'un discours. En tant que discours, elle dépendra de $N$. Ainsi, la justification de $N$ dépendra de l'utilisation et de l'acceptation de N. Mais alors, cette justification sera nécessairement circulaire. Ainsi, non seulement notre « rapport » aux normes de base est problématique, mais leur justification (qui fait partie elle aussi d'une bonne explication de notre rapport aux normes) l'est au moins tout autant - sinon plus.

Pour ces raisons, je suis d'accord avec Mulligan lorsqu'il dit que notre rapport aux normes épistémiques est «difficile à expliquer». Ce rapport demeure une énigme, et je pense que c'est ce que Wittgenstein tente d'élucider dans De la certitude. C'est aussi ce que les penseurs de la tradition austro-allemande comme Ortega et d'autres (notamment Husserl), dont je n'ai pas pu discuter ici, tentent de faire. Cela s'inscrit dans une démarche qui a pour but de rendre compte de la condition humaine, au sens où notre rapport aux normes est une composante essentielle de ce que nous sommes. L'ouvrage de Mulligan contribue, en comparant diverses traditions et en mettant côte-à-côte certains textes, à mettre en lumière toute la richesse de cette démarche. 